\title{
Examen coproparasitológico en niños: comparación de resultados obtenidos por dos métodos en dos instituciones de Santafé de Bogotá, D.C.
}

\author{
Duque S. ${ }^{1}$, Guerrero R. ${ }^{2}$, Nicholls R.S. ${ }^{3}$, López M.C. ${ }^{4}$
}

\section{RESUMEN}

Con el fin de comparar la frecuencia de hallazgo de parásitos intestinales en niños, mediante el examen directo realizado en una entidad hospitalaria de tercer nivel, el Hospital Infantil Lorencita Villegas de Santos (HILVS) y en una institución de referencia, el Instituto Nacional de Salud (INS), Grupo de Parasitología, y confrontarlo con el método de concentración en formol-éter practicado en el INS, se tomaron al azar 487 muestras de materia fecal de las 2.954 recibidas en el laboratorio de la entidad hospitalaria durante un período de seis meses. Estas muestras fueron fraccionadas para su remisión al laboratorio de parasitología de la entidad de referencia.

Posteriormente, se revisaron las historias clínicas de 305 de los 398 niños cuyas muestras (coprológicos seriados) fueron analizadas, para describir las variables demográficas y las posibles indicaciones de solicitud del examen. Sin embargo, éstas no representaron necesariamente la sintomatología asociada con los parásitos.

Se encontraron $277(56,9 \%)$ muestras negativas y $210(43,1 \%)$ positivas para por lo menos un parásito, siendo 93 de ellas $(19,1 \%)$ positivas para algún patógeno.

El grado de concordancia entre la entidad hospitalaria y la institución de referencia se determinó utilizando el índice Kappa. La concordancia en la identificación de los parásitos fue casi perfecta con Giardia lamblia, notable con Trichomonas hominis, moderada con lodamoeba bütschlii y Entamoeba coli, regular con Endolimax nana, Trichuris trichiura y Blastocystis hominis, mala con Entamoeba histolytica y nula con Entamoeba hartmanni.

La comparación de los valores de sensibilidad y especificidad obtenidos de la evaluación del examen directo y el método de concentración formol-éter del examen coproparasitológico, mostró una mayor detección de parásitos con el método de concentración. Sin embargo, por la superposición de los valores del límite superior de la sensibilidad del primero (directo) con los del límite inferior de sensibilidad del segundo (concentración), no pudo establecerse la existencia de esta ventaja.

\footnotetext{
Bióloga, MSc parasitología médica. grupo de parasitología, Instituto Nacional de Salud. Santafé de Bogotá, Colombia. 2 Médico cirujano, MSc gastroenterología pediátrica. Jefe, Servicio de Gastroenterología, Hospital Infantil Lorencita Villegas de Santos, Santafé de Bogotá, Colombia.

3 Médico cirujano, MSc parasitología médica. Jefe Grupo de parasitología, Instituto Nacional de Salud. Profesor adscrito, Departamento de microbiología y parasitología, Facultad de medicina, Universidad Nacional de Colombia, Santafé de Bogotá, Colombia.

4 Bacterióloga, Grupo de parasitología, Instituto Nacional de Salud, Santafé de Bogotá, Colombia.
} 
La prevalencia de las diversas parasitosis fue menor que la encontrada en la Encuesta Nacional de Morbilidad de Parasitismo Intestinal.

Se hace énfasis en la necesidad de una solicitud justificada del examen para evitar costos innecesarios, así como en la importancia de los adecuados procedimientos diagnósticos y de establecer criterios morfológicos precisos para un correcto diagnóstico de laboratorio.

\section{SUMMARY}

This survey was carried out in order to compare the frequency of intestinal parasite detection by direct wet film in stool specimens from children done simultaneously at the Hospital Infantil Lorencita Villegas de Santos (HILVS) and at the Parasitology Department of the Instituto Nacional de Salud (INS). The usefulness of two methods for the detection of intestinal parasites in the same institution (INS) was compared direct wet examination and concentration by the formalin-ether sedimentation technique,

Four hundred and eighty seven faecal samples of children were taken randomly from 2,954 received at HILVS during six months. A fraction of each sample was sent from the hospital to INS for its parasitological examination.

Later on, 305 out of 398 clinical records belonging to children whose stools had been examined every day during three consecutive days, were retrospectively reviewed to describe some demographic factors and the possible signs and symptoms which necessitated the stool examination. The signs and symptoms found by the revision of the clinical records did not necessarily correspond to those associated with intestinal parasites.

No parasites were detected in two hundred and seventy seven $(56.9 \%)$ samples. Of the two hundred and ten $(43.1 \%)$ positive samples, pathogenic parasites were found in ninety three (19.1\%).

The concordance between the examination of faeces at HILVS and INS was measured by the Kappa index. The strength of agreement was almost perfect for Giardia lamblia, substantial for Trichomonas hominis, moderate for lodamoeba bütschliiand Entamoeba coli, fair for Endolimax nana, Trichuris trichiura and Blastocystis hominis, slight for Entamoeba histolytica and poor for Entamoeba hartmanni.

The sensitivity and specificity values obtained from direct wet examination and the formalin-ether sedimentation technique showed a greater identification of parasites by the concentration method. Nevertheless, there is overlapping between the upper limit of the confidence interval for the direct wet mount and the lower limit of the confidence interval for the formalin-ether sedimentation technique. It is therefore not possible to conclude that the sensitivity of the concentration method is better than that of the direct wet mount.

The overall and specific prevalences of parasites were lower than those reported by the National morbidity survey on intestinal parasitism.

The study emphasizes the following topics: 1) the need to set up guidelines and precise indications for ordering faecal examinations in order to avoid loss of time and money; 2) the importance of the accuracy of the diagnostic methods, and 3) the need to establish precise morphological criteria for the correct identification of parasites at any laboratory. 
El parasitismo intestinal constituye un problema de salud pública particularmente en los países en vía de desarrollo por sus deficientes condiciones de higiene y saneamiento ambiental. Su frecuencia y manifestaciones clínicas pueden ser particularmente severas en niños.

El diagnóstico de las parasitosis intestinales se establece en general por el hallazgo de formas parasitarias en el examen directo de materia fecal. Sin embargo, también puede realizarse por métodos de concentración que, de acuerdo con estudios previos (1-4), presentan una mayor sensibilidad.

El presente trabajo tuvo por objeto comparar el método directo realizado en una entidad hospitalaria (Hospital Lorencita Villegas de Santos) y dos métodos diferentes (directo y concentración formol-éter) practicados en un laboratorio de referencia (Grupo de Parasitología, Instituto Nacional de Salud), con muestras de materia fecal procedentes de una población infantil hospitalaria de Santafé de Bogotá, y establecer la prevalencia de parásitos intestinales en ella.

\section{Materiales y métodos}

Muestras: de dos mil novecientos cincuenta y cuatro muestras de materia fecal procesadas en el laboratorio central de la entidad hospitalaria, durante junio-noviembre de 1991, se tomaron 487 al azar sin orden secuencial para el análisis ciego e independiente por parte de los dos laboratorios. Estas no fueron seleccionadas por los investigadores, quienes tampoco participaron en la solicitud del examen coprológico.

Las muestras fueron recolectadas y fraccionadas en el laboratorio central de la entidad hospitalaria, entre 7-10 a.m. y remitidas al laboratorio de parasitología de la institución de referencia en las primeras horas de la tarde (1-2 p.m.) donde se procesaron en cuanto fueron recibidas.

Se consideraron positivas aquellas muestras que lo fueran para cualquier parásito por cualquiera de los métodos.

\section{Métodos}

Examen coproparasitológico: en la entidad hospitalaria se llevó a cabo el examen directo en solución salina fisiológica y en coloración con lugol. En el laboratorio de parasitología de la institución de referencia se realizaron dos métodos: 1) directo, tanto en solución salina fisiológica como en coloración con lugol, 2) concentración por sedimentación con formol-éter.

\section{Examen directo en solución salina fisiológica}

- Se colocó una gota de solución salina fisiológica a la izquierda de una lámina portaobjeto.

- Se tomó con la punta de un aplicador materia fecal de diferentes partes de la muestra hasta completar una cantidad pequeña.

- Se homogenizó la muestra perfectamente en la gota de solución salina fisiológica.

- Se cubrió la preparación con una laminilla de $22 \times 22 \mathrm{~mm}$.

- Se observó al microscopio en objetivo 10X.

- Se examinó sistemáticamente toda el área bajo la laminilla .

Cuando se observó algo que morfológicamente era semejante a un agente parasitario, se examinó el especimen en objetivo de $40 \mathrm{X}$ (5).

\section{Examen directo, coloración con lugol}

- Se colocó una gota de lugol a la derecha de una lámina portaobjeto.

- Se tomó con la punta de un aplicador materia fecal de diferentes partes de la muestra hasta completar una cantidad pequeña.

- Se homogenizó la muestra perfectamente en la gota de lugol.

- Se cubrió la preparación con una laminilla de $22 \times 22 \mathrm{~mm}$.

- Se examinó al microscopio como se describió anteriormente.

\section{Concentración formol-éter}

- Sevirtieron $12 \mathrm{ml}$ de formol al $10 \%$ en un vaso plástico pequeño desechable.

- Se agregaron $2 g$ de materia fecal.

- Se homogenizó perfectamente la muestra. 
- Se filtró el homogenizado a través de gasa doble.

- Se transfirió el filtrado a un tubo de centrífuga.

- Se agregaron 2-3 ml de éter etílico al filtrado obtenido.

- Se agitó vigorosamente la mezcla durante 12 minutos.

- Se centrifugó a 1000 g durante 5 minutos.

- Se desprendió, con un escobillón, el tapón formado por los residuos presentes en la materia fecal centrifugada, de las paredes del tubo.

- Se eliminó el sobrenadante.

- Se mezcló el sedimento.

- Se colocó una gota del sedimento en el centro de una lámina portaobjeto.

- Se agregó una gota de lugol.

- Se homogenizó la preparación perfectamente en la gota de lugol.

- Se cubrió la preparación con una laminilla de $22 \times 22 \mathrm{~mm}$.

- Se examinó al microscopio $(6,7)$.

Revisión de historias clínicas: se revisaron retrospectivamente las historias clínicas de ios pacientes cuyas muestras fueron analizadas, para registrar las variables demográficas (sexo, edad, procedencia), el estado nutricional y los datos clínicos que presumiblemente sirvieron como indicación para el examen coproparasitológico.

Procesamiento y análisis de la información: la tabulación de la información se realizó en el programa Epi-info (8).

La concordancia en la identificación de los parásitos entre el examen directo realizado en la entidad hospitalaria y el efectuado en el laboratorio de la institución de referencia, se estableció utilizando el índice Kappa (9).

Para cada uno de los exámenes realizados, se determinó la sensibilidad definiendo ésta como el cociente del número de muestras encontradas positivas por ese método, para un determinado parásito, dividido por el número de muestras positivas para éste por cualquiera de los métodos (10).
Para cada valor de sensibilidad y especificidad se establecieron los intervalos de confianza mediante las tablas científicas del documento Geigy (11) y los valores de sensibilidad, de los diferentes métodos para cada uno de los parásitos, fueron comparados.

\section{Resultados}

Las 487 muestras correspondían a 398 pacientes de quienes se pudo revisar, mediante búsqueda retrospectiva, la información clínica de 305.

\section{Descripción de la población:}

Las edades oscilaron entre 2 meses y 18 años; 167 pacientes $(54,7 \%)$ de sexo masculino y 138 $(45,3 \%)$ femenino. La distribución por edad y sexo se encuentra en la tabla 1.

El 94,1\% (287) de los pacientes provenía de zona urbana y solamente el 5,9\% (18) de área rural.

En relación con el estado nutricional, 6,0\% de los niños se encontraba con relación peso/talla en percentil menor o igual a tres, $17,1 \%$ menor o igual a diez y el $67,8 \%$ de la población se encontraba entre los percentiles 10 y 90 de acuerdo con las tablas de National Center for Health Statistics (NCHS). El estado nutricional se encuentra detallado en la tabla 2.

Los síntomas que sirvieron como probable indicación del examen coproparasitológico se presentan en la tabla 3; los más frecuentes fueron dolor abdominal $(42,3 \%)$, diarrea aguda $(30,8 \%)$ y vómito $(20,3 \%)$. Es de anotar que no se encontró la información clínica pertinente en el 16,4\% de las historias clínicas revisadas.

Tabla 1. Distribución por edad y sexo de la población estudiada

\begin{tabular}{lrrrr}
\hline \multicolumn{1}{r}{ Edad } & \multicolumn{3}{c}{ Sexo } \\
\multicolumn{1}{r}{ (años) } & M & F & Total & $\%$ \\
\hline Menor de 1 & 11 & 14 & 25 & 8,20 \\
$1-5$ & 80 & 62 & 142 & 46,60 \\
$6-10$ & 50 & 41 & 91 & 29,80 \\
$11-15$ & 24 & 19 & 43 & 14,10 \\
Mayor de 15 & 2 & 2 & 4 & 1,30 \\
\hline & & & & \\
TOTAL & 167 & 138 & 305 & 100,00
\end{tabular}


Tabla 2. Estado nutricional de la población estudiada

\begin{tabular}{lcc}
\hline $\begin{array}{l}\text { Peso/talla * } \\
\text { Percentil }\end{array}$ & Frecuencia & Porcentaje \\
\hline Menor de 3 & 17 & 6,0 \\
Menor de 10 & 49 & 17,1 \\
$10-90$ & 194 & 67,8 \\
Mayor de 90 & 9 & 3,1 \\
Mayor de 97 & 17 & 6,0 \\
\hline & & \\
Total & 286 & 100,0 \\
\hline
\end{tabular}

* National Center for Health Statistics

Tabla 3. Probable indicación de examen coprológico

\begin{tabular}{lrr}
\hline Síntomas & no.* & $\%$ \\
\hline Dolor abdominal & 129 & 42,3 \\
Diarrea aguda & 94 & 30,8 \\
Vómito & 62 & 20,3 \\
Anorexia & 27 & 8,9 \\
Diarrea crónica & 19 & 6,2 \\
Retardo en el crecimiento & 18 & 5,9 \\
Rectorragia & 6 & 2,0 \\
Distensión abdominal & 2 & 0,7 \\
Eosinofilia & 2 & 0,7 \\
Flatulencia & 1 & 0,3 \\
Náuseas & 1 & 0,3 \\
Astenia & 1 & 0,3 \\
Anemia & 1 & 0,3 \\
Heces grasosas & 1 & 0,3 \\
Sin información & 50 & 16,4 \\
\hline
\end{tabular}

(*) Número de pacientes $=305$

\section{Examen coproparasitológico}

De las 487 muestras analizadas, 277 (56,9\%) fueron negativas para cualquier parásito en los métodos utilizados y solamente en $93(19,1 \%)$ se encontró uno o más parásitos patógenos (tabla 4). Los protozoos considerados patógenos presentaron una frecuencia del $6,2 \%$ y $9,2 \%$ para $E$. histolytica y $G$. lamblia respectivamente. La frecuencia de los helmintos fue muy baja, siendo el Ascaris lumbricoides el más frecuente $(1,8 \%)$. Las frecuencias de los demás parásitos se encuentran en la tabla 5 .
La concordancia en la identificación de parásitos mediante el examen directo realizado en los dos laboratorios se registra en la tabla 6 . La concordancia con respecto a la determinación de parásitos y de acuerdo las convenciones establecidas para los valores obtenidos del índice Kappa (9) fue nula para $E$. hartmanni, mala para $E$. histolytica, regular para Blastocystis hominis, Endolimax nana y Trichuris trichiura, moderada para lodamoeba bütschlii y E. coli, notable para Trichomonas hominis y casi perfecta para $G$. lamblia.

Tabla 4. Frecuencia de muestras positivas y negativas

\begin{tabular}{|c|c|c|}
\hline & No. & $\%$ \\
\hline $\begin{array}{l}\text { Muestras negativas para } \\
\text { cualquier parásito }\end{array}$ & 277 & 56,9 \\
\hline Muestras positivas $\left({ }^{\star}\right)$ & 210 & 43,1 \\
\hline $\begin{array}{l}\text { Muestras negativas para } \\
\text { parásitos considerados } \\
\text { patógenos }\left({ }^{\star \star}\right) \text {. }\end{array}$ & 117 & 24,0 \\
\hline $\begin{array}{l}\text { Muestras positivas para, } \\
\text { por lo menos, un patógeno }\end{array}$ & 93 & 19,1 \\
\hline
\end{tabular}

${ }^{*}$ ) Se consideran positivas aquellas muestras en las que se encontró al menos un parásito, patógeno o no.

$\left.{ }^{(\star}\right)$ Se consideraron como patógenos los siguientes: Entamoeba histolytica, Giardia lamblia, Ascaris lumbricoides, Trichuris trichiura y uncinarias.

Tabla 5. Parasitismo intestinal Frecuencia en 487 muestras

\begin{tabular}{lrr}
\hline Parásito & No. & $\%$ \\
\hline & 30 & 6,2 \\
Entamoeba histolytica & 6 & 1,2 \\
Entamoeba hartmanni & 79 & 16,2 \\
Entamoeba coli & 79 & 16,2 \\
Endolimax nana & 17 & 3,5 \\
lodamoeba bütschlii & 45 & 9,2 \\
Giardia lamblia & 2 & 0,4 \\
Chilomastix mesnili & 43 & 13,1 \\
Blastocystis hominis & 2 & 0,4 \\
Trichomonas hominis & 8 & 1,6 \\
Trichuris trichiura & 9 & 1,8 \\
Ascaris lumbricoides & 2 & 0,4 \\
Uncinarias & &
\end{tabular}


Tabla 6. Examen directo. Grado de concordancia (índice KAPPA). Hospital Infantil Lorencita Villegas de Santos vs. Instituto Nacional de Salud.

\begin{tabular}{lcl}
\hline Parásito & Valor de Kappa & Concordancia \\
\hline Entamoeba hartmanni & 0,00 & Nula \\
Entamoeba histolytica & 0,22 & Mala \\
Blastocystis hominis & 0,25 & Regular \\
Trichuris trichiura & 0,33 & Regular \\
Endolimax nana & 0,39 & Regular \\
lodamoeba bütschlii & 0,41 & Moderada \\
Entamoeba coli & 0,43 & Moderada \\
Trichomonas hominis & 0,67 & Notable \\
Giardia lamblia & 0,81 & Casi perfecta \\
\hline
\end{tabular}

Interpretación de los valores de Kappa:

$\begin{aligned} \text { Kappa } & \text { Concordancia } \\ <0 & \text { Nula } \\ 0-0,20 & \text { Mala } \\ 0,21-0,40 & \text { Regular } \\ 0,41-0,60 & \text { Moderada } \\ 0,61-0,80 & \text { Notable } \\ 0,81-1,00 & \text { Casi perfecta }\end{aligned}$

Los valores de sensibilidad y especificidad obtenidos para la identificación de parásitos por los dos métodos se informan en la tabla 7. La especificidad tuvo los mismos valores en la determinación de especímenes realizando cualquiera de los métodos.

Se encontraron diferencias en los valores de sensibilidad para el diagnóstico de diferentes parásitos por un mismo método como también para la determinación de un mismo parásito por diferentes métodos. Los valores menores se obtuvieron por el método directo para E. hartmanni, E. coli, E. nana, I. bütschlii, G. lamblia, B. hominis, $T$. trichiura y uncinarias. Sin embargo, las diferencias obtenidas no fueron significativas ya que existe superposición de los intervalos de confianza (tabla 7).

Los parásitos intestinales patógenos más frecuentes en la población estudiada fueron $E$. histolytica y G. lamblia (tabla 8) siendo ésta más prevalente, a diferencia de las helmintiasis causadas por uncinarias, $A$. lumbricoides y $T$. trichiura

Tabla 7. Comparación de sensibilidad y especificidad entre los métodos coprológico directo (1) y concentración (2)

\begin{tabular}{|c|c|c|c|c|c|c|c|c|c|}
\hline Parásito & método & $\mathrm{n}$ & $\mathrm{S}$ & LIS & LSS & $n$ & E & LIE & LSE \\
\hline \multirow[t]{2}{*}{ E. histolytica } & 1 & 30 & 0,27 & 0,12 & 0,45 & 457 & 1,00 & 0,99 & 1,00 \\
\hline & 2 & 30 & 0,27 & 0,12 & 0,45 & 457 & 1,00 & 0,99 & 1,00 \\
\hline \multirow[t]{2}{*}{ E. hartmanni } & 1 & 6 & 0,67 & 0,22 & 0,95 & 481 & 1,00 & 0,99 & 1,00 \\
\hline & 2 & 6 & 1,00 & 0,54 & 1,00 & 481 & 1,00 & 0,99 & 1,00 \\
\hline \multirow[t]{2}{*}{ E. coli } & 1 & 79 & 0,53 & 0,41 & 0,64 & 408 & 1,00 & 0,99 & 1,00 \\
\hline & 2 & 79 & 0,72 & 0,61 & 0,81 & 408 & 1,00 & 0,99 & 1,00 \\
\hline \multirow[t]{2}{*}{ E. nana } & 1 & 79 & 0,44 & 0,33 & 0,56 & 408 & 1,00 & 0,99 & 1,00 \\
\hline & 2 & 79 & 0,61 & 0,49 & 0,71 & 408 & 1,00 & 0,99 & 1,00 \\
\hline \multirow[t]{2}{*}{ I bütschlii } & 1 & 17 & 0,35 & 0,14 & 0,61 & 470 & 1,00 & 0,99 & 1,00 \\
\hline & 2 & 17 & 0,53 & 0,28 & 0,77 & 470 & 1,00 & 0,99 & 1,00 \\
\hline \multirow[t]{2}{*}{ G. lamblia } & 1 & 45 & 0,78 & 0,66 & 0,90 & 442 & 1,00 & 0,99 & 1,00 \\
\hline & 2 & 45 & 0,91 & 0,83 & 0,99 & 442 & 1,00 & 0,99 & 1,00 \\
\hline \multirow[t]{2}{*}{ C. mesnili } & 1 & 2 & 1,00 & 0,15 & 1,00 & 485 & 1,00 & 0,99 & 1,00 \\
\hline & 2 & 2 & 1,00 & 0,15 & 1,00 & 485 & 1,00 & 0,99 & 1,00 \\
\hline \multirow[t]{2}{*}{ B. hominis } & 1 & 64 & 0,22 & 0,12 & 0,32 & 423 & 1,00 & 0,99 & 1,00 \\
\hline & 2 & 64 & 0,53 & 0,40 & 0,66 & 423 & 1,00 & 0,99 & 1,00 \\
\hline \multirow[t]{2}{*}{ A. lumbricoides } & 1 & 9 & 1,00 & 0,66 & 1,00 & 478 & 1,00 & 0,99 & 1,00 \\
\hline & 2 & 9 & 1,00 & 0,66 & 1,00 & 478 & 1,00 & 0,99 & 1,00 \\
\hline \multirow[t]{2}{*}{ T. trichiura } & 1 & 8 & 0,25 & 0,03 & 0,65 & 479 & 1,00 & 0,99 & 1,00 \\
\hline & 2 & 8 & 0,50 & 0,15 & 0,85 & 479 & 1,00 & 0,99 & 1,00 \\
\hline \multirow[t]{2}{*}{ Uncinarias } & 1 & 2 & 0,00 & 0,00 & 0,84 & 485 & 1,00 & 0,99 & 1,00 \\
\hline & 2 & 2 & 1,00 & 0,15 & 1,00 & 485 & 1,00 & 0,99 & 1,00 \\
\hline
\end{tabular}

$S$ : sensibilidad

E : especificidad

$\mathrm{n}$ : número de muestras
LIS : límite inferior de la sensibilidad

LIE : límite inferior de la especificidad
LSS : límite superior de la sensibilidad

LSE : límite superior de la especificidad 
Tabla 8. Prevalencias (\%) de Entamoeba histolytica y Giardia lamblia, por grupos de edad.

\begin{tabular}{|c|c|c|c|c|}
\hline \multirow[t]{2}{*}{ Edad (años) } & \multicolumn{2}{|c|}{ E. histolytica } & \multicolumn{2}{|c|}{ G. lamblia } \\
\hline & $\begin{array}{c}\text { Población } \\
\text { estudiada } \\
1991\end{array}$ & $\begin{array}{c}\text { Encuesta Nal. } \\
\text { Morbilidad } \\
1980^{*}\end{array}$ & $\begin{array}{c}\text { Población } \\
\text { estudiada } \\
1991\end{array}$ & $\begin{array}{c}\text { Encuesta Nal. } \\
\text { Morbilidad } \\
1980^{\star}\end{array}$ \\
\hline Menor de 1 & 4 & 2,5 & 0 & 13,4 \\
\hline $1-4$ & 0,1 & 8,7 & 14,0 & 31,1 \\
\hline $5-14$ & 8,1 & 14,8 & 10,6 & 21,7 \\
\hline Mayor de 15 & 16,6 & $10,8 * *$ & 0 & 9,3 \\
\hline
\end{tabular}

* Corredor A. Parasitismo intestinal. Encuesta Nacional de Morbilidad 1980. (En prensa).

* En la Encuesta Nacional de Morbilidad está definido de 15 a 24 años.

cuyas prevalencias fueron relativamente bajas, teniendo en cuenta las prevalencias informadas en la Encuesta Nacional de Morbilidad de 1980 (12).

No se encontró asociación significativa entre positividad para Giardia lamblia y Entamoeba histolytica y el estado nutricional.

\section{Discusión}

Las técnicas de concentración de quistes, huevos y larvas, presentes en una muestra de materia fecal, ya sea por métodos de flotación $(4,13)$ o de concentración, han demostrado una sensibilidad mayor con respecto a aquellos procedimientos donde se analiza la muestra directamente $(1,2,4)$ con solución salina (13) o lugol (5). Aunque nuestros resultados mostraron un incremento en la eficiencia diagnóstica del concentrado, puesto que el mayor número de parásitos fue determinado por el método de concentración formol-éter al compararlo con el examen directo realizado en el mismo laboratorio de la institución de referencia, la superposición de los valores del límite superior de la sensibilidad del examen directo con los del límite inferior del método de concentración, no nos permite recomendar en forma absoluta la utilización rutinaria del método de concentración para mejorar la calidad del diagnóstico clínico. Assefa en 1991 (14) obtuvo un resultado similar al comparar ambas metodologías para el diagnóstico de un sólo helminto (Strongyloides stercolaris). Es probable que desde el punto de vista netamente parasitológico, no clínico, el rendimiento diagnóstico sea mayor con la utilización secuencial de los dos métodos, directo y concentración formol-éter.

Por otra parte, la utilización del directo permite, además, la observación de trofozoítos los cuales no pueden ser observados con el procedimiento de concentración formol-éter $u$ otras metodologías de sedimentación (15) en las cuales se utilicen químicos, pues éstos los destruyen.

Las diferencias obtenidas en la determinación de parásitos entre los directos, especialmente en el grupo de amibas, podrían explicarse desde varios puntos de vista. En primer lugar, por diferencias en los criterios para la identificación de las amibas.

Es frecuente la incorrecta identificación de trofozoítos de E. histolytica como macrófagos, hecho observado por Walsh en 1986 (16). La motilidad y emisión de seudópodos pueden prestarse a confusión en la identificación si no se posee la suficiente experiencia y no se realiza la tinción con azul de metileno que permite una diferenciación clara.

Es importante anotar que, además, pueden ocurrir errores en la identificación de las formas quísticas. Los quistes de E. histolytica y E. hartmannison morfológicamente iguales, hecho que podría explicar la incorrecta identificación de quistes de la segunda como quistes de la primera, si no se realiza una medición de éstos para definirlas, como ocurrió en la entidad hospitalaria. La medición es un factor importante en la identificación de las amibas ya que éstas, por su grado de madurez, pueden presentar desde 1 hasta 4 núcleos en $E$. histolytica y $E$. hartmanni y desde 1 hasta 8 en E. coliy, por ende, diferencia en tamaños, permitiendo ubicar en los límites de medición a una $E$. coli dentro de E. histolytica si no se observan con detalle las demás estructuras internas como los cuerpos cromatoides que éstos poseen.

En segundo lugar, por el prolongado período transcurrido entre la recolección de la muestra y el examen de ésta en la institución de referencia. Los trofozoítos, especialmente los de E. histolytica, son de corta viabilidad en la materia fecal una vez emitida $(17,18)$. Las muestras fueron analizadas 
independientemente por las entidades con un intervalo de 6-8 horas. Es posible que este factor fuese uno de los contribuyentes a la gran diferencia entre las frecuencias de $E$. histolytica observadas. Sin embargo, en la institución hospitalaria solamente se observaron trofozoítos de $E$. histolytica en un caso, razón por la cual, podría descartarse esta explicación.

En tercer lugar, las diferencias podrían ser debidas también, al hecho de que en la entidad de referencia sólo intervinieron dos personas en el diagnóstico coproparasitológico, mientras que en la hospitalaria participaron siete personas, lo cual indudablemente aumenta la variabilidad en la interpretación diagnóstica.

El grado regular de concordancia en el caso de E. nana podría explicarse desde el punto de vista de la observación morfológica de los quistes. Algunas veces se pueden confundir con levaduras.

La gran variedad morfológica que posee el $B$. hominis permite que estructuras seudo-parasitarias sean determinadas como tales.

La presencia de helmintos en las materias fecales examinadas fue baja y ello quizás se deba a las características de la población en sí, pues la mayoría provenía de zona urbana, Santafé de Bogotá, D.C., donde las condiciones ambientales no son propicias para el desarrollo en el suelo de las geohelmintiasis.

La muestra estudiada representa únicamente la población de una entidad hospitalaria; por lo tanto, sus características y las frecuencias observadas no son extrapolables a la población general.

Primordialmente se trata de población urbana, bien nutrida en, al menos, el $80 \%$, que consultó a una entidad de tercer nivel. La poca presencia de niños provenientes de zonas rurales puede explicar en parte la diferencia en relación con los datos de la Encuesta Nacional de Morbilidad (12). En protozoos como G. lamblia se observa una mayor tasa en preescolares, patrón previamente observado en dicha encuesta. La infección por $E$. histolytica muestra una tendencia a incrementar con la edad. Debido al bajo número de muestras encontradas positivas para helmintos, no es posible establecer comparaciones con los resultados de la Encuesta Nacional de Morbilidad.

La información obtenida de las historias clínicas como indicación presuntiva para la solicitud de los coprológicos no representa necesariamente la sintomatología asociada con los parásitos. La clínica de las parasitosis es bastante inespecífica. Además, el interrogatorio no fue dirigido para todos los síntomas registrados. Los más frecuentes fueron dolor abdominal, diarrea aguda y vómito. El primero puede tener múltiples explicaciones en las edades pediátricas, si bien puede indicar presencia de algunos parásitos, en especial G. lamblia, lo que justificaría la solicitud de los coprológicos; los dos últimos pueden corresponder a síntomas de gastroenteritis de diversa etiología, la más frecuente de origen viral (19), lo que podría explicar el número alto de exámenes negativos. Esto recuerda, indirectamente, que el médico debe preocuparse por solicitar el examen cuando se justifique, si proporciona información útil para el diagnóstico y manejo del paciente sin incrementar innecesariamente los costos.

Los hallazgos más sobresalientes de este estudio fueron: 1) el alto número de muestras negativas; 2) los diferentes grados de concordancia en la identificación de parásitos utilizando el examen directo; 3) el rendimiento diagnóstico puede ser mayor con la utilización secuencial de los dos métodos, desde el punto de vista netamente parasitológico, no clínico; y 4) la mayor frecuencia de E. histolytica en el examen directo en la institución hospitalaria. Estos hallazgos plantean inquietudes sobre la real necesidad de los exámenes solicitados y sobre la posibilidad de que exista un exceso de diagnóstico de E. histolytica en la institución hospitalaria, lo que podría generar formulación innecesaria de medicamentos amebicidas.

Es necesario enfatizar que el diagnóstico de las parasitosis en los niños puede comprobarse con un diagnóstico de laboratorio confiable que tenga en cuenta la recolección correcta, el procesamiento oportuno y la conservación adecuada de las muestras; la toma múltiple dentro de una misma muestra; la utilización del micrómetro ocular para la medición de quistes y la unificación 
de criterios morfológicos precisos. Esto es particularmente valedero para las entidades hospitalarias donde no existe personal dedicado exclusivamente al diagnóstico parasitológico y es usual la rotación de personal, tanto calificado como en proceso de aprendizaje y entrenamiento en el manejo y lectura de exámenes coprológicos.

\section{Agradecimientos}

Los autores agradecen especialmente al doctor Juan Manuel Lozano por su revisión crítica al trabajo, al doctor Luis Carlos Orozco Vargas por su asesoría en el manejo de información y análisis estadístico y al personal del Laboratorio del Hospital Infantil Lorencita Villegas de Santos: Carmen Cecilia Trujillo, jefe, y a las siguientes bacteriólogas quienes realizaron el examen directo de las materias fecales: María Consuelo Osorio, Adriana Calle, Constanza Vanegas, Margarita Franco, María Gladys Oviedo, Cecilia Almeira y Nelly Espinosa.

\section{Referencias}

1. Zierdt WS. A simple device for concentration of parasite eggs, larvae, and protozoa. Am J Clin Pathol 1978; 70: 89-93.

2. McMillan A, McNeillage GJC. Comparison of the sensitivity of microscopy and culture in the laboratory diagnosis of intestinal protozoal infection. J Clin Pathol 1984; 37 : 809-11.

3. Truant AL, Elliot SH, Kelly MY, Smith JH. Comparison of formalin ethylether sedimentation, formalin-ethylacetate sedimentation, and zinc sulfate flotation techniques for detection of intestinal parasites. J Clin Microbiol 1981; 13: $882-4$

4. Perry JL, Mathews JS, Miller GR. Parasite detection efficiencies of five concentration systems. J Clin Microbiol 1990; 28: 1094-7.

5. Manson-Bahr PEC, Bell DR. Manson's tropical diseases. 19thed. London: Bailliere Tindall.1987: 1489-90.
6. Ridley DS, Hawgood BC. The value of formol-ether concentration of faecal cysts and ova. J Clin Pathol 1956; 9: 74-6.

7. Allen AVH, Ridley DS. Further observations on the formol-ether concentration technique for faecal parasites. $J$ Clin Pathol 1970; 23: 545-6.

8. Dean AG, Dean JA, Burton AH, Decker RC. Epi-Info, versión 5: a word processing, database, and statistics program for epidemiology on microcomputers. Atlanta: Center for Disease Control, 1990.

9. Kramer MS, Fenistein AR. Clinical biostatistics. LIV. The biostatistics of concordance. Clin Pharmacol Ther 1981; 29: 111-23.

10. Grimer PF, Mayewski RJ, Mushlin Al, Greenland P. Selection and interpretation of diagnostic tests and procedures. Ann Intern Med 1981; 94: 553-600.

11. Geigy JR, S.A. ed. Documento Geigy. Tablas científicas. Basilea, Suiza, 6a ed.

12. Corredor A. Parasitismo intestinal. Encuesta Nacional de Morbilidad. 1980. (En prensa).

13. Faust EC, D'Antoni JS, Odom V, et al. A critical study of clinical laboratory technics for the diagnosis of protozoan cysts and helminth eggs in feces. Am J Trop Med 1938; 18: $169-83$.

14. Assefa T, Woldemichael T, Seyoum T. Evaluation of the modified Baermann's method in the laboratory diagnosis of Strongyloides stercolaris. Ethiop Med J 1991; 29: 193-8.

15. Long EG, Tsin AT, Robinson BA. Comparison of the FeKal CON-Trate system with the formalin-ethyl acetate technique for detection of intestinal parasites. J Clin Microbiol 1985; 22: 210-1.

16. Walsh JA. Problems in recognition and diagnosis of amebiasis: estimation of the global magnitude of morbidity and mortality. Rev Infec Dis 1986; 8: 228-38.

17. Brooke MM, Goldman M. Polyvinyl alcohol-fixative as preservative and adhesive for protozoa in dysenteric stools and other liquid materials. J Lab Clin Med 1949; 34: 1554-60.

18. Scholten TH, Yang J. Evaluation of unpreserved and preserved stools for the detection and identification of intestinal parasites. Am J Clin Pathol 1974; 62: 563-7.

19. Leal FJ, Franco G, Rodríguez G, Córdoba F, Rodríguez A. Malnutrition and etiological agents of acute diarrhea. International Pediatr, 1990; 5: 142. 